\section{ROGAVF STUDY 2019 - Relationship of HbA1C (GLYCEMIC Control) on outcomes of AV FISTULAS: A prospective observational study}

\author{
Premjeet Singh" ${ }^{1 *}$ Zainal Ariffin ${ }^{1}$, Hafizan Tajri' ${ }^{1}$ Azim Md Idris², \\ Lenny Suryati ${ }^{2}$ and Lily Sofida ${ }^{1}$
}

1Department of Vascular and General Surgery, Hospital Kuala Lumpur, Malaysia

${ }^{2}$ Department of Vascular Surgery, Hospital University Kebangsaan, Malaysia

\begin{abstract}
Objective: The main aim of the study was to compare outcomes based on diabetic control for patients undergoing formation of a new upper limb arteriovenous fistula (AVF). Research design and methods: A prospective cohort study was performed where we obtained baseline $\mathrm{HbA} 1 \mathrm{c}$ in 65 patients before undergoing AV fistula formation. Patients were followed up at our clinic 6 weeks after creation to assess fistula maturity.
\end{abstract}

Results: Multiple logistic regression was used to analyze the association between $\mathrm{HbA} 1 \mathrm{c}$ status and maturity of AVF at 6 weeks after controlling for possible confounding factors such as age, sex, presence of hypertension and dyslipidaemia. Those with $\mathrm{HbA} 1 \mathrm{c}$ less than 6.5 were 22 times likely to have maturity of AVF at 6 weeks as compared to those with HbA1c 6.5 or more (AOR $=22.65, p<0.005$ )

Conclusion: Good diabetes control, reflected by an $\mathrm{HbA} 1 \mathrm{c}$ of less than 6.5 , is associated with a very high possibility of AVF maturity at 6 weeks post creation.

\section{More Information}

\begin{abstract}
*Address for Correspondence: Premjeet
Singh, Department of Vascular and General Surgery, Hospital Kuala Lumpur, Malaysia, Tel: +60122490377;

Email: premjeet.singh@hotmail.com

Submitted: 15 July 2019

Approved: 19 July 2019

Published: 22 July 2019

How to cite this article: Singh P, Ariffin Z, Tajri H, Md Idris A, Suryati L, et al. ROGAVF STUDY 2019 - Relationship of HbA1C (GLYCEMIC Control) on outcomes of AV FISTULAS: A prospective observational study. Arch Surg Clin Res. 2019; 3: 053-055
\end{abstract}

DOI: dx.doi.org/10.29328/journal.ascr.1001033

Copyright: @ 2019 Singh P, et al. This is an open access article distributed under the Creative Commons Attribution License, which permits unrestricted use, distribution, and reproduction in any medium, provided the original work is properly cited

Check for updates

\section{Introduction}

Diabetes is a leading cause of kidney failure and has an increasing prevalence globally [1]. Diabetic patients with end-stage renal failure requiring renal replacement therapy in the form of hemodialysis are best managed with an autologous arteriovenous fistula (AVF), which is regarded as the optimum form of vascular access [2]. Diabetic patients are predisposed to a higher incidence of peripheral vascular disease than people without diabetes, with poorer flow rates and a heavier burden of arterial wall calcification [3].

In view of majority of ESRF patients requiring dialysis are diabetics in this country, this should not be a reason to dissuade them from fistula creation. However, if good control of diabetes before creation of fistula is associated with better outcome, we can optimize our patients before the surgery and this can lead to better outcome. This study is designed to see if there is an association between good diabetes control before surgery and rate of maturity in 6 weeks' time.

\section{Research Design and Methodology}

This prospective observational cohort study was conducted in Hospital Kuala Lumpur for a period of 15 months. The study population in this study was all diabetics going for first AV fistula creation. Universal sampling was done, where all diabetics for AVF creation were recruited during the study period. Only patients that were diabetic and planned for brachiocephalic fistula were recruited. Other inclusion criteria were, vein size of at least $2 \mathrm{~mm}$ and it must be the first AVF created in the patient. Patients that were on erythropoietin supplements and pediatric patients were excluded. All diabetic patients, which fit into the inclusion and exclusion criteria, had an $\mathrm{HbA1c}$ test taken on the day of fistula creation. HbA1c reflects diabetes control in the past 2 months and an HbA1c level taken on the day of creation will reflect the control of diabetes before the fistula is created [4]. Demographic data such as age, sex and ethnicity, as well as presence of comorbids such as hypertension, and dyslipidaemia were collected as well. All the patients 
underwent a brachiocephalic creation by the same vascular surgeon using the same technique (prolene 6/0 continuous sutures for the anastomosis). Patients were discharged the same day after the procedure and seen in the clinic after 6 weeks. A duplex scan was performed to assess fistula maturity. Any complications arising from AVF creation were treated as per standard medical procedure. Maturation of fistula (primary end-point) is measured using the "rule of $6 s$ " that describes parameters associated with maturity of a newly created AV fistula as set forth by the Kidney Disease Outcomes Quality Initiative (KDOQI) guidelines for dialysis access [5]. The rule of $6 \mathrm{~s}$ states that 6 weeks after the AV fistula has been placed, the fistula is more likely to be usable if: the external diameter is greater than $0.6 \mathrm{~cm}$, the fistula is no more than $0.6 \mathrm{~cm}$ deep from the skin surface, and blood flow rate is greater than $600 \mathrm{~mL} /$ minute, All data that was collected was tabulated and computerized in MS Excel and was analyzed using SPSS version 23.0. Patients were divided into 2 groups. One with HbA1c of $>6.5$ and the other with HbA1c of $<6.5$. Comparison between outcomes of AVF (Successfully mature at 6 weeks) was analyzed using multiple logistic regression, adjusting dyslipidemia, hypertension, age, sex and ethnicity.

\section{Results}

A total of 65 patients we recruited in this study. The demographic and descriptive data of the patients enrolled in this study are as shown on table 1 . The mean age of the study population was 54.7 ( $\mathrm{SD}=12.6$ ) years. Almost $59 \%$ of them were males and rest were females. Majority of the patients were Malay $(72 \%)$ they had dyslipidaemia or hypertension ( $81 \%$ and $72 \%$ respectively). $73 \%$ of all Brachiocephalic fistulas created matured in 6 weeks. About 7\% had delayed maturity, meaning fistula matured eventually but not in 6 weeks. A small percentage had surgical site infection and cellulitis.

Multiple logistic regression was used to analyse the association between $\mathrm{HbA1c}$ status and maturity of AVF at 6 weeks after controlling for possible confounding factors. Those with HbA1c less than 6.5 were 22 times more likely to have maturity of AVF at 6 weeks as compared to those with HbA1c 6.5 or more $(\mathrm{AOR}=22.65,95 \% \mathrm{CI}=2.56-200.34$ ). Hypertension has been excluded from the data analysis due to significant interaction with dyslipidaemia (Table 2).

The association between HbA1c and complications were determined using Fisher's exact test. There was no significant association between HbA1c status and delayed maturity ( $p$ $=0.388$ ). There is also no association between surgical site infection and cellulitis between the two cohorts $(\mathrm{p}=1.00$ for both) as shown in table 3 .

\section{Discussion}

Angiopathy is a known complication of long-standing diabetes. Many patients in Malaysia with end stage renal disease have a background of diabetes. Performing a vascular

\begin{tabular}{|c|c|c|}
\hline & & $N=65$ \\
\hline Age & Mean (SD) & $54.7(12.6)$ \\
\hline \multirow{2}{*}{ Gender } & \multirow{2}{*}{$\begin{array}{c}\text { Male } \\
\text { Female }\end{array}$} & 38 (58.5) \\
\hline & & $27(41.5)$ \\
\hline \multirow{3}{*}{ Ethnicity } & \multirow{3}{*}{$\begin{array}{l}\text { Malary } \\
\text { Chinese } \\
\text { Indian }\end{array}$} & $47(72.3)$ \\
\hline & & $7(10.8)$ \\
\hline & & $11(16.9)$ \\
\hline \multirow{2}{*}{ Medical history } & \multirow{2}{*}{$\begin{array}{l}\text { Hypertension } \\
\text { Dyslipidemia }\end{array}$} & $53(81.5)$ \\
\hline & & $47(72.3)$ \\
\hline \multirow{3}{*}{ HbA1c } & \multirow{3}{*}{$\begin{array}{c}\text { Mean (SD) } \\
\quad<6.5 \\
>6.5\end{array}$} & $6.9(1.8)$ \\
\hline & & $27(41.5)$ \\
\hline & & $38(58.5)$ \\
\hline Fistula maturity & - & $48(73.8)$ \\
\hline \multirow{3}{*}{ Complication } & \multirow{3}{*}{$\begin{array}{c}\text { Delayed maturity } \\
\text { SSI } \\
\text { Cellutis }\end{array}$} & $6(9.2)$ \\
\hline & & $1(1.5)$ \\
\hline & & $2(3.1)$ \\
\hline
\end{tabular}

Table 2: Relationship between HbA1c and maturity of AVF in 6 weeks using logistic regression controlling for age, ethnicity, hypertension and dyslipidaemia

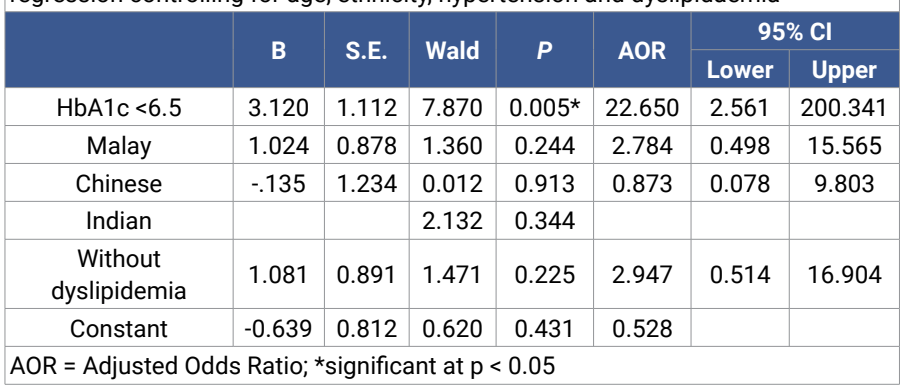

Table 3: Comparison of difference in complications between study groups.

\begin{tabular}{|c|c|c|c|c|}
\hline \multirow{2}{*}{ Complication } & \multicolumn{4}{|c|}{ HbA1c } \\
\hline \multirow{2}{*}{ Delayed maturity } & No & $26(96.3)$ & $33(86.8)$ & 0.388 \\
\hline & Yes & $1(3.7)$ & $5(13.2)$ & \\
\hline SSI & No & $27(100.0)$ & $37(97.4)$ & 1.000 \\
\hline & Yes & $0(0.0)$ & $1(2.6)$ & \\
\hline Cellutis & No & $26(96.3)$ & $37(97.4)$ & 1.000 \\
\hline & Yes & $1(3.7)$ & $1(2.6)$ & \\
\hline
\end{tabular}

access surgery or any vascular surgery in a diabetic patient often yield unsatisfactory results. Historically, diabetic patients who undergo AVFs fared worse than their nondiabetic counterparts, and this may have been misconceived as a reason to exclude diabetic patients from receiving a potential AVF and to offer them alternative forms of vascular access [6]. When it comes to previous published literature, there has been conflicting results. Mortaz et al. in a paper published in Urology Journal in 2013, conducted a retrospective study comparing factors influencing fistula outcome and noted no difference in the patency rate during the first 2 years [7]. Gordon et al. in 2016, also retrospectively studied the outcome (primary failure rate and time taken to mature) between diabetics and non-diabetics and it showed no significant difference [6]. On the contrary, studies by Sesso (1995 in Brazil), Tuka (2012) and Miller (1999) all shows the fistulas either matures later, has less patency rates and has higher risk of primary failure [8-10]. All these studies are retrospective. 
When it comes to diabetes control, $\mathrm{Wu}$, published remarkable results for patients with good diabetes control with HbA1c of less than 7, who underwent angioplasty of AVF compared to diabetics with an HbA1c of more than 7. His data showed the probability of good diabetes control leading to a successful angioplasty of an AV fistula of being as high as $61 \%$ as compared to only $30 \%$ in non-diabetic [11].

Based on our study, with good control of diabetes, chances of a fistula to mature is significantly better. Other factors have been attributed to predicting fistula outcome. Increasing age and female gender has been attributed to poor outcome along with diabetes mellitus [12]. In our study, these confounding factors, along with dyslipidaemia and ethnicity were eliminated using statistical test and diabetes control alone was analysed.

Diabetes mellitus is known to cause atherosclerosis or varying degree in medium and small size arteries, especially when diabetes is poorly controlled. Medium and small arteries are used for upper limb arteriovenous fistula creation. It is postulated that impaired flow in these arteries, due to atherosclerosis, inhibit physiological dilation of the artery following an arteriovenous anastomosis creation, which in turn impedes venous dilation and maturation.

Complications of diabetes mellitus in vascular access surgery such as delayed maturity, surgical site infection and cellulitis were more common in the poorly controlled diabetics as compared to the other group, but it is statistically not significant. This could be attributed to our small sample size. In our study, a single experienced surgeon performed all the fistulas. This eliminates surgeon to surgeon bias in terms of experience and surgical technique.

\section{Conclusion}

This study was carefully planned and performed with an adequate sample size which demonstrates a clear difference between fistula maturity at 6 weeks between the 2 cohorts. Patients who are diabetics should not be dissuaded from having an AVF created but the procedure should only be performed when patient's diabetes status is well controlled as it proven to be associated with better outcome.

\section{References}

1. Shaw JE, Sicree RA, Zimmet PZ. Global estimates of the prevalence of diabetes for 2010 and 2030. Diabetes Res Clin Pract. 2010; 87: 4-14. PubMed: https://www.ncbi.nlm.nih.gov/pubmed/19896746

2. Fluck R, Kumwenda M. UK Renal Association Clinical Practice Guidelines on vascular access for haemodialysis. Nephron Clin Pract. 2011; 118: c225-240.

PubMed: https://www.ncbi.nlm.nih.gov/pubmed/21555898

3. Malovrh M. Native arteriovenous fistula: preoperative evaluation. Am J Kidney Dis. 2002; 39: 1218-1225.

PubMed: https://www.ncbi.nlm.nih.gov/pubmed/12046034

4. Byori R. Indicators of glycemic control --hemoglobin A1c (HbA1c), glycated albumin (GA), and 1,5-anhydroglucitol (1,5-AG). Rinsho Byori. 2014; 62: 45-52.

PubMed: https://www.ncbi.nlm.nih.gov/pubmed/24724426

5. Andrew C Gordon, et al. Diabetes should not disuade aretriovenous fistula formation. British Journal of Diabetes. 2016.

6. Mortaz AV. Fistulas in Diabetics. Urology Journal. 2013; 10: 826-827.

7. Sesso R, Melaragno CS, Luconi PS, et al. [Survival of dialyzed diabetic patients]. Rev Assoc Med Bras. 1995; 41: 178-8214.

8. Asif A, Roy-Chaudhury P, Beathard GA. Early arteriovenous fistula failure: a logical proposal for when and how to intervene. Clin $\mathrm{J} \mathrm{Am}$ Soc Nephrol. 2006; 1: 332-339.

PubMed: https://www.ncbi.nlm.nih.gov/pubmed/17699225

9. Tuka V, Slavikova M, Svobodova J, Malik J. Diabetes and distal access location are associated with higher wall shear rate in feeding artery of PTFE grafts. Nephrol Dial Transplant. 2006; 21: 2821-2824. PubMed: https://www.ncbi.nlm.nih.gov/pubmed/16735379

10. Miller PE, Tolwani A, Luscy CP, Deierhoi MH, Bailey R, et al. Predictors of adequacy of arteriovenous fistulas in hemodialysis patients. Kidney Int. 1999; 56: 275-280.

PubMed: https://www.ncbi.nlm.nih.gov/pubmed/10411703

11. Wu CC, Wen SC, Yang CW, Pu SY, Tsai KC, et al. Baseline plasma glycemic profiles but not inflammatory biomarkers predict symptomatic restenosis after angioplasty of arteriovenous fistulas in patients with hemodialysis. Artherosclerosis. 2010; 209: 598-600. PubMed: https://www.ncbi.nlm.nih.gov/pubmed/19939386

12. Murphy GJ, Nicholson ML. Autogeneous elbow fistulas: the effect of diabetes mellitus on maturation, patency, and complication rates. Eur J Vasc Endovasc Surg. 2002; 23: 452-457.

PubMed: https://www.ncbi.nlm.nih.gov/pubmed/12027475 